\title{
Desenvolvimento de um Ingrediente Alimentar Funcional: CaracterizaÇão por AnIQUilação do Positrão
}

\author{
M.F. Ferreira Marques ${ }^{1,2}$, C.I.C. Galhano ${ }^{3,4}$ e A. Moreira da Silva ${ }^{3,5, *}$
}

\begin{abstract}
0
interesse dos consumidores numa alimentação saudável tem vindo a crescer à medida que vão sendo divulgados os potenciais benefícios para a saúde de determinados alimentos e/ou ingredientes alimentares. As evidências científicas apoiam a ideia de que alguns alimentos, ou ingredientes alimentares, possam ter efeitos positivos sobre a saúde e bem-estar, para além do fornecimento dos nutrientes básicos. Alguns projetos de investigação já levaram à identificação e compreensão dos mecanismos dos componentes alimentares biologicamente ativos que podem reduzir o risco de doença. Neste contexto, é introduzido o conceito de Alimento Funcional. O principal objetivo do estudo aqui relatado consistiu na caracterização de um potencial ingrediente alimentar funcional, a timoquinona, um componente ativo, obtido a partir de um óleo essencial de sementes de Nigella sativa. De forma a aumentar a biodisponibilidade da timoquinona, foi utilizada uma estratégia que consistiu na síntese de um sistema de nanopartículas. Entre as várias moléculas existentes capazes de formar essas estruturas (supramoleculares), as ciclodextrinas têm vindo a ser utilizadas de forma a aumentar a solubilidade de moléculas de natureza hidrófoba. A espectroscopia de tempos de vida de Aniquilação do Positrão (PALS -"Positron Annihilation Lifetime Spectroscopy") foi usada na caraterização do composto de inclusão baseado em $\beta$-ciclodextrina e timoquinona. A apresentação deste estudo suscita a referência a outros desafios que se apresentam à comunidade científica para a investigação de alimentos funcionais, de forma a entender-se os mecanismos potenciais dos componentes ativos dos alimentos que poderão melhorar o estado de saúde e de bem-estar e, possivelmente, reduzir o risco de doença.
\end{abstract}

Alimentos Funcionals: O ÓleO ESSENCIAL DE NIGELLA SATIVA E A TIMOQUINONA COMO POTENCIAIS INGREDIENTES ALIMENTARES FUNCIONAIS

No projeto de investigação iniciado, o principal objetivo foi o desenvolvimento de um potencial ingrediente alimentar funcional. Este desenvolvimento foi precedido pela caracterização do constituinte principal do óleo essencial de Nigella sativa (Figura 1 (a) - planta medicinal com usos ancestrais), a timoquinona (Figura 1 (d)).

A primeira questão que se pode colocar sobre este tema é: "O que são Alimentos Funcionais"? Na tentativa de responder identificam-se uma série de questões com definição... "Qualquer alimento pode ser funcional?", é uma das questões que surge imediatamente. "O que define um Alimento Funcional? Existem muitas definições para alimentos funcionais, mas não há nenhuma definição ofi-

\footnotetext{
Instituto Superior de Engenharia de Coimbra, 3031-199 Coimbra

CEMUC, Departamento de Física, Universidade de Coimbra, 3004-516 Coimbra

Escola Superior Agrária de Coimbra, Bencanta, 3040316 Coimbra

Unidade de investigação CERNAS, Bencanta, 3040316 Coimbra

Unidade de investigação Química-Física Molecular, Universidade de Coimbra, 3000-535 Coimbra

*E-mail: aidams@esac.pt
}

cial que seja universalmente aceite. Um ponto de vista mais redutor é que qualquer alimento é de facto funcional, porque tem a função de fornecer nutrientes e possui efeitos fisiológicos. Nesse contexto, o termo Alimento Funcional poderá ser considerado um termo de marketing, para um alimento cuja atração reside nas reivindicações de saúde e da forma como o produto é entendido pelo consumidor. Há quem defenda que, qualquer alimento, quando comercializado adequadamente, acompanhado por uma alegação de efeitos positivos sobre a saúde, é um Alimento Funcional.

Alguns alimentos considerados como Alimentos Funcionais são realmente alimentos naturais integrais, onde podem ser usadas informações científicas atualizadas sobre os efeitos na saúde para anunciar benefícios. Muitos, se não a maioria, são frutas, legumes, grãos, peixes, laticínios e produtos cárnicos. Estes alimentos contêm vários componentes naturais que poderão proporcionar efeitos benéficos para a saúde, para além da nutrição básica. Alguns exemplos incluem o licopeno (Figura 2) do tomate processado, os ácidos gordos $\omega 3$ (Figura 3) do salmão ou as saponinas (Figura 4) da soja. Mesmo o chá e o chocolate têm sido seguidos nalguns estudos, sendo-lhes atribuído propriedades funcionais, ou seja, atributos com efeitos positivos para a saúde e bem estar geral para além da nutrição básica (Tabela 1).

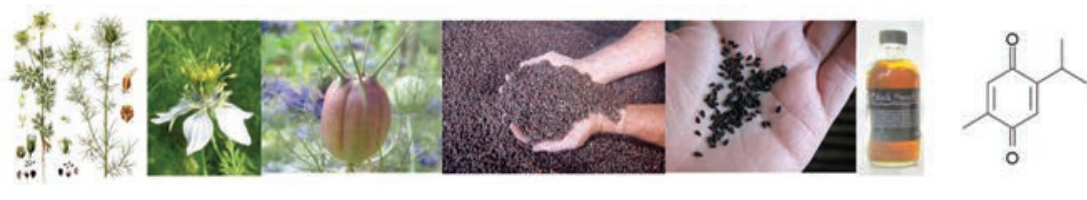

(a)

(b)

(c)

(d)

Figura 1 - Nigella sativa (a), sementes de cominhos-negros (b), óleo essencial (c) e molécula de timoquinona (d)

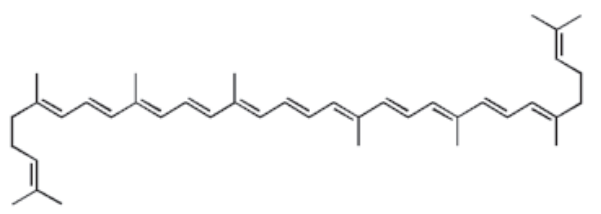

Figura 2 - Estrutura molecular do licopeno 


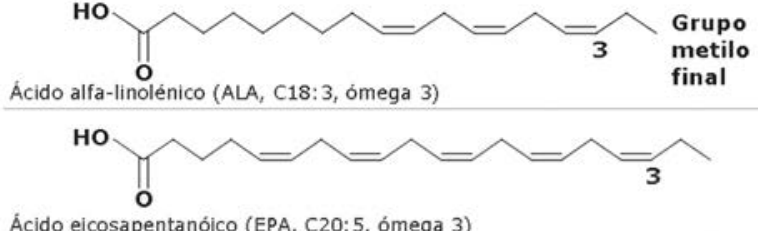

Ácido eicosapentanóico (EPA, C20:5, ómega 3)<smiles>CCC=CCC=CCC=CCC=CCC=CCC=CCCC(=O)O</smiles>

Ácido docosahexanóico (DHA, C22:6, ómega 3)

HO<smiles>CCCCCC=CCC=CCCCCCCCC(C)=O</smiles>

Ácido linoleico (LA, C18:2, ómega 6)

HO<smiles>CCCCCC=CCC=CCC=CCC=CCCCC(C)=O</smiles>

Ácido araquidónico (AA, C20:4, ómega 6)

Figura 3 - Ácidos gordos $\omega 3$ e $\omega 6$

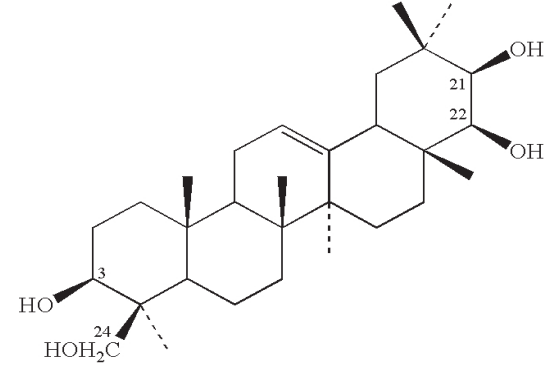

Figura 4 - Estrutura molecular de uma saponina, heterosídeo do metabolismo secundário vegetal, apresentando propriedades tensioativas

Tabela 1 - Exemplos de Alimentos Funcionais e/ou Componentes Funcionais, fonte alimentar e respetivos benefícios potenciais [2]

\begin{tabular}{|c|c|c|}
\hline Componentes Funcionais & Fonte & Beneficios potenciais \\
\hline \multicolumn{3}{|l|}{ Prebióticos/Probióticos } \\
\hline Frutoligosacáridos (FOS) & Helianthus tuberosus, alho, cebola em pó & Efeitos positivos sobre a microflora intestinal \\
\hline Lactobacillus casei immunitas & Leite fermentado, outros laticínios & Melhoramento da saúde gastrointestinal \\
\hline \multicolumn{3}{|l|}{ Carotenoides } \\
\hline $\begin{array}{l}\text { Alfa-caroteno } \\
\text { Beta-caroteno }\end{array}$ & Cenouras, frutos vegetais & Aniquilação de radicais livres \\
\hline Luteína & Vegetais verdes & Redução do risco de degeneração da mácula \\
\hline Licopeno & Tomate (ketchup, molhos) & Redução do risco de cancro da próstata \\
\hline \multicolumn{3}{|l|}{ Fibra } \\
\hline Fibra Insolúvel & Farelo de trigo & $\begin{array}{l}\text { Redução do risco de cancro da mama e do } \\
\text { cólon }\end{array}$ \\
\hline Beta-Glucana & Aveia, cevada & \multirow{2}{*}{$\begin{array}{l}\text { Redução do risco de doença cardiovascular. } \\
\text { Redução do colesterol }\end{array}$} \\
\hline Fibra Solúvel & Psyllium & \\
\hline \multicolumn{3}{|l|}{ Ácidos Gordos } \\
\hline Ácidos gordos ómega -3 (DHA/EPA) & Salmão e outros óleos de peixe & $\begin{array}{l}\text { Redução do risco de doença cardiovascular. } \\
\text { Melhora as funções cognitivas e visuais }\end{array}$ \\
\hline Ácido Linoleico Conjugado (CLA) & Queijo, carnes & $\begin{array}{c}\text { Melhora a composição corporal. Reduz o risco } \\
\text { de doenças }\end{array}$ \\
\hline \multicolumn{3}{|l|}{ Compostos Fenólicos } \\
\hline Antocianidinas & Frutos & \multirow{4}{*}{$\begin{array}{l}\text { Neutraliza os radicais livres e reduz o risco de } \\
\text { cancro }\end{array}$} \\
\hline Catequinas & Chá & \\
\hline Flavononas & Citrinos & \\
\hline Flavonas & Frutos/vegetais & \\
\hline Lignanos & Centeio, vegetais & Prevenção de cancro e de falhas renais \\
\hline Taninos (proantocianidinas) & Arando, cacau, chocolate & $\begin{array}{l}\text { Melhora a saúde do trato urinário. Reduz o } \\
\text { risco de doença cardiovascular }\end{array}$ \\
\hline \multicolumn{3}{|l|}{ Fitoesteróis } \\
\hline Éster de Estanol & Milho, soja, trigo & $\begin{array}{l}\text { Redução dos níveis de colesterol sanguíneo, } \\
\text { através da redução da absorção do colesterol }\end{array}$ \\
\hline \multicolumn{3}{|l|}{ Fitoestrogénios de Soja } \\
\hline Isoflavonas & Grãos de soja e alimentos com base de soja & $\begin{array}{l}\text { Alívio dos sintomas adversos da menopausa. } \\
\text { Redução dos níveis de colesterol sanguíneo }\end{array}$ \\
\hline
\end{tabular}

Há ainda quem defenda que só um alimento que foi intencionalmente fortificado, enriquecido ou reforçado com determinado componente que possui determinado benefício acrescido para a saúde, deverá ser considerado como funcional. A maioria das definições também sugere que um alimento funcional deve ser, ou parecer, um alimento tradicional e deve fazer parte da nos- sa dieta normal. Um alimento funcional pode ser dirigido à totalidade da população ou a grupos específicos, que podem ser definidos, por exemplo, por idade ou por constituição genética [1]. 
Regressando ao sistema em estudo, a timoquinona (TQ), principal constituinte de sementes de Nigella sativa (Figura 1 (b) - sementes negras), importa referir que tem sido tradicionalmente empregue na medicina popular, devido a vários efeitos farmacológicos. Vários estudos recentes relatam que a TQ exibe fortes efeitos anti-oxidantes, anti-inflamatórios, anti-neoplásicos e analgésicos, tanto in vitro como in vivo [1].

Atualmente existe um interesse crescente no potencial terapêutico da TQ em diferentes áreas de investigação médica, incluindo os estudos sobre a diabetes, mas, particularmente, na terapia de diversas formas de cancro. A TQ é um potente inibidor em células do cancro do cólon, de leucemia, do carcinoma da laringe, do cancro pancreático, do adernocarcinoma do ovário, do sarcoma uterino e do cancro da próstata, embora apresente uma baixa toxicidade para células não neoplásicas. Para além disso, a TQ e a planta medicinal Nigella sativa são também promissores agentes quimiopreventivos [1].

A maioria dos fármacos, especialmente os de natureza hidrófoba, falha nos ensaios clínicos em humanos, devido à eficácia reduzida, que pode ser, em parte, uma consequência da sua baixa biodisponibilidade. Embora os produtos naturais tenham suscitado um grande interesse da comunidade científica, a maioria dos fármacos de origem natural utilizados clinicamente apresentam uma baixa biodisponibilidade. Esta desvantagem tem dificultado a aplicação prática. Uma estratégia para a resolução da desvantagem anteriormente descrita tem sido a inclusão de moléculas de natureza hidrófoba em ciclodextrinas (CDs), com o objetivo de produzir preparações mais estáveis e biodisponíveis [3].
As CDs são oligossacarídeos cíclicos, macrociclos, com uma superfície hidrófila e uma porção interna de natureza hidrófoba, que consiste em seis $(\alpha-C D)$, sete $(\beta-C D)$, ou oito ( $\gamma$ $C D)$ anéis de D-glucopiranose unidos através de ligações glicosídicas do tipo a-1,4 (Figura $5(\mathrm{a})$ ), que podem ser representados como uma estrutura de cone truncado, ou de "donut" molecular (Figura 5 (b)). Os diâmetros da cavidade interior são aproximadamente 0,57, 0,78 e 0,95 nm, respetivamente (Figura 5 (c)). As vantagens fundamentais de ciclodextrinas naturais como veículos de fármacos e/ ou ingredientes funcionais, são as seguintes: (i) a estrutura química é conhecida, com vários locais potenciais para modificações químicas ou por conjugação, (ii) a disponibilidade de diferentes tamanhos de cavidades, (iii) a sua baixa toxicidade e atividade farmacológica, (iv) a solubilidade em água, (v) a proteção contra a biodegradação dos conjugados de fármacos e/ou ingredientes alimentares incluídos [3].

A molécula da $\beta$-ciclodextrina é uma forma muito utilizada em sistemas alimentares, tendo sido aprovada em 1998 para a utilização em géneros alimentícios, constando na lista Europeia dos Aditivos Alimentares com a referência E-459. Por outro lado, a lista de moléculas convidadas (guests) disponíveis para encapsulação em ciclodextrinas inclui compostos tais como hidrocarbonetos de cadeia linear ou ramificada, aldeídos, cetonas, álcoois, ácidos orgânicos, ácidos gordos, compostos aromáticos, gases (por exemplo, 1-metilciclopropeno, cu-

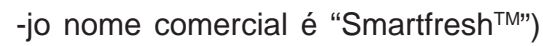
e compostos polares, tais como halogéneos e oxiácidos aminas. A cavidade hidrófoba da ciclodextrina fornece um ambiente de modo a que moléculas não polares de tamanho adequado

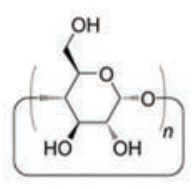

(a)

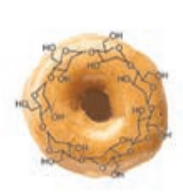

(b)

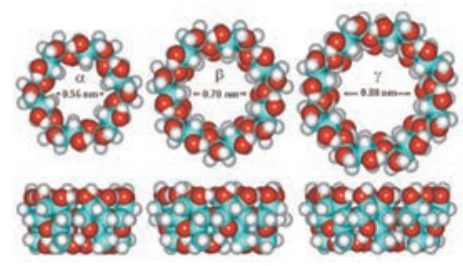

(c)

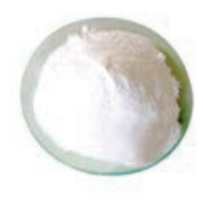

(d)

Figura 5 - Estrutura do oligossacárido cíclico, ciclodextrina, CD (a) n igual a seis, sete ou oito anéis de D-glicopiranose, unidos através de ligações glicosídicas do tipo $\alpha-1,4$ (b), representação de $\alpha-C D, \beta-C D$ e $\gamma-C D(c)$, respetivamente. Pó branco cristalino de $\beta-C D(d)$

possam entrar e formar espécies de inclusão estáveis. Durante a inclusão são quebradas ou formadas ligações do tipo não covalente. Uma das aplicações mais importantes de ciclodextrinas nas áreas farmacêutica e alimentar é a melhoria da solubilidade aquosa das moléculas através do processo de inclusão, criando estruturas supramoleculares ${ }^{1}$.

O MÉTODO EXPERIMENTAL E OS MATERIAIS USADOS: A TÉCNICA DE ANIQUILAÇÃO DO POSITRÃO, A TIMOQUINONA E A $\beta$-CICLODEXTRINA

O eletrão, e-, e a sua antipartícula, o positrão, $\mathrm{e}^{+}$, são partículas fundamentais que apresentam as seguintes características: sendo partículas de spin $1 / 2$, obedecem à estatística de Fermi-Dirac e verificam o princípio de Pauli; das quatro interações conhecidas na natureza (gravítica, eletromagnética, fraca e forte), só não participam diretamente na interação forte.

Pondo de lado os positrões presentes na radiação cósmica, os positrões que se encontram na natureza são em geral o produto de um decaimento radioativo do tipo $\beta^{+}$(resultado de interação fraca).

O positrão, que no vazio é uma partícula estável, pode aniquilar-se na matéria ao encontrar um eletrão. O positrão pode também ligar-se com um eletrão para formar um átomo do tipo do hidrogénio, o positrónio (Ps), que assume um de dois estados diferentes: o estado singuleto, ${ }^{1} \mathrm{~S}_{0}$, designado por para-positrónio ( $p$-Ps), com os spins do positrão e do eletrão em orientações opostas, e o estado tripleto, ${ }^{3} \mathrm{~S}_{1}$, o orto-positrónio (o-Ps), cujos dois spins têm a mesma orientação.

As probabilidades de formação destes dois estados são de aproximadamente $25 \%$ para o $p$-Ps e $75 \%$ para o o-Ps.

Uma vez que o Ps é um átomo do tipo do hidrogénio, o seu tratamento em mecânica quântica é muito semeIhante ao do próprio hidrogénio, mas com algumas diferenças substanciais. Contrariamente aos átomos, para os quais o modelo clássico de Bohr permite imaginar um núcleo central à vol- 
ta do qual gravitam os eletrões, para o Ps é difícil uma tal representação. Pode-se contudo imaginá-lo como um sistema de duas partículas que gravitam à volta do seu centro de massa, e cujas identidades são perdidas em proveito de uma entidade neutra, pelo que o seu comportamento é semeIhante ao de um átomo do tipo hidrogenóide, muito leve.

Existem três processos diferentes de decaimento do positrão e/ou do positrónio. Num dado meio condensado corresponderão um tempo de vida, $\tau_{\mathrm{i}}$, e uma abundância relativa, $l_{i}$, característicos. De um modo geral, num meio homogéneo condensado, revelam-se três componentes cujas vidas médias, $\tau_{i}$, e respetivas abundâncias relativas (intensidades), $l_{\mathrm{i}}$, estão associadas às várias espécies positrónicas, com os índices $\mathrm{i}=1-3$ a corresponderem ao $p$-Ps (125 ps), e+ livre (300-500 ps) e o-Ps (alguns nanosegundos), respetivamente.

Uma vez formado o positrónio, ele pode interagir com um eletrão do meio que possua um spin de sentido oposto ao do seu próprio eletrão, resultando daí a aniquilação. Tanto o o-Ps como o $p$-Ps podem sofrer aniquilação por pick-off. Nestas condições, é afinal a vida média do o-Ps que vai ser significativamente modificada por tal processo, reduzindo-se de 140 ns (no vazio) a alguns ns, enquanto o efeito sobre o $p$-Ps é menos importante dado a sua vida média ser muito mais curta.

Uma vez injetados num meio condensado, os positrões provenientes do decaimento de um núcleo radioativo vão perdendo progressivamente a sua energia cinética inicial (tipicamente de umas centenas de keV) através de ionizações, excitações (eletrónicas, vibracionais e rotacionais) e múltiplos choques elásticos. Após este processo de termalização, ou o positrão se aniquila espontaneamente com um eletrão, ou forma o positrónio, Ps. A grande importância do o-Ps justifica-se por ser uma sonda das mais adequadas à determinação das propriedades físico-químicas da matéria. O sucesso da aplicação de PALS (espectroscopia de tempos de vida da aniquilação do positrão - Positronium Annihilation Lifetime Spec- troscopy) resulta do facto de o átomo de positrónio na matéria se localizar preferencialmente em volumes livres da ordem de grandeza de dimensões moleculares, ficando aí aprisionado e sujeito às forças repulsivas provocados pelas nuvens eletrónicas das moléculas envolventes. Este comportamento resulta na capacidade da técnica de PALS fornecer valores confiáveis para as dimensões e frações de volume livre.

As dimensões dos "buracos" e a fração de volume livre são grandezas importantes para a caracterização micro e macroestrutural de materiais, uma vez que é possível correlacionar a informação (sub) microscópica com propriedades macroscópicas [4]. A técnica que tem por base a aniquilação de positrões utiliza nuclídeos radioativos emissores $\beta^{+}$, e na espectroscopia de tempos de vida, a fonte usada é de ${ }^{22} \mathrm{Na}$. O esquema de decaimento do ${ }^{22} \mathrm{Na}$ está representado na Figura 6 (a), onde se vê que a quase totalidade dos decaimentos $\beta^{+}$conduz ao estado excitado do ${ }^{22} \mathrm{Ne}$, que decai para o estado fundamental com emissão dum fotão $\gamma$ de 1,2746 MeV. Sendo o tempo de vida deste estado muito curto (3 ps), quando comparado com o dos positrões em líquidos ou materiais poliméricos, o fotão de 1,2746 MeV é usado como referência para o instante de criação do positrão, Figura 6 (b).

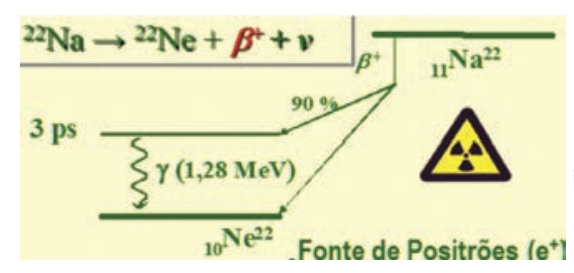

Figura 6 (a) - Esquema do decaimento da fonte de ${ }^{22} \mathrm{Na}$
A fonte mais comum na espectroscopia de tempos de vida em sólidos tem uma estrutura de sandwich. O material radioativo é depositado numa folha de kapton $^{\circledR}$, sobre a qual, após secagem, é colocada uma outra folha do mesmo material, sendo o conjunto, por fim, devidamente selado. Tipicamente, as amostras para este estudo são duas pastilhas com espessura suficiente, colocadas justapostas à fonte, uma de cada lado.

O sistema utilizado compreende dois detetores de radiação gama $(\gamma)$. Um deles deteta o fotão de 1,2746 MeV, que identifica o instante em que o positrão é produzido (start), e o outro deteta um dos fotões de $511 \mathrm{keV}$, resultante da aniquilação (stop). Os detetores, constituídos por cintilador plástico lido por fotomultiplicador, são os componentes mais críticos para a otimização da resolução e da eficiência dum espectrómetro de tempo. Além do divisor de tensão para o fotomultiplicador, as bases incluem a eletrónica que faz a seleção da janela de energia, sendo os sinais de tempo tirados dum dínodo do fotomultiplicador, Figura 7.

A técnica da espectroscopia de tempos de vida assenta na determinação da distribuição temporal de intervalos de tempo entre a criação (start) e aniquilação (stop) de positrões. A utilização da fonte radioativa de ${ }^{22} \mathrm{Na}$

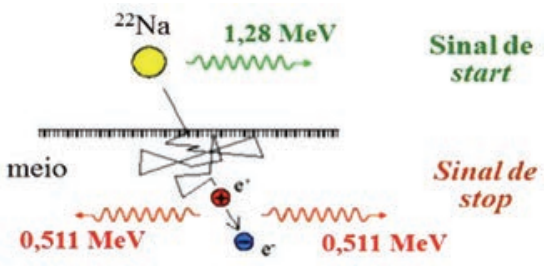

Figura 6 (b) - Esquema da fonte de ${ }^{22} \mathrm{Na}$ e da interação da radiação emitida com o meio

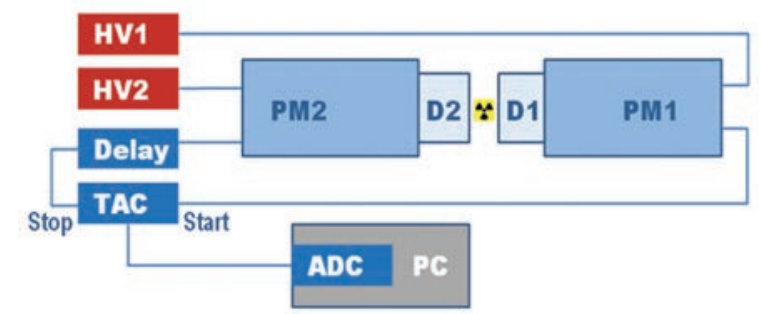

Figura 7 - Esquema do sistema usado para o registo dos espectros de tempo. PM1 e PM2 fotomultiplicadores; D1 e D2 cintiladores; HV1 e HV2 - fontes de alimentação; Delay - atraso do sinal de stop; TAC (Time to Amplitude Converter) - conversor de tempo em amplitude, que transforma o intervalo de tempo entre a chegada dos sinais de start e de stop num sinal cuja amplitude é proporcional ao valor desse intervalo de tempo; ADC (Analog to Digital Converter) - conversor analógico-digital 
torna fácil essa medida, uma vez que a produção do positrão é acompanhada pela emissão de um fotão $y$ de energia suficientemente afastada dos 511 keV para que seja possível a sua identificação sem ambiguidade pelo sistema experimental.

O espectro de tempos de vida observado experimentalmente, $N(t)$, é uma soma de funções exponenciais (uma por cada "espécie" positrónica) convoluída com a função de resolução do sistema experimental, $R(t)$, e sobreposta ao fundo, F. Na Figura 8, apresenta-se um espectro típico de uma amostra com os três tempos de vida.

No estudo aqui relatado usaram-se os compostos $\beta-C D$, oferta da Wacker-Chemie, e TQ que foi adquirida à Sigma. Os compostos de inclusão foram preparados pelo método de co-precipitação [3].

Cada amostra, em pó, de $\beta$-CD com inclusão de TQ, foi sujeita a uma pressão de $\sim 70$ bar para formar discos de $10 \mathrm{~mm}$ de diâmetro e cerca de $0,5 \mathrm{~mm}$ de espessura. A fonte de positrões ${ }^{22} \mathrm{Na}$ (ca. $7 \times 10^{4} \mathrm{~Bq}$, encerrada entre folhas de Kapton ${ }^{\circledR}$ ), foi colocada entre dois discos de amostras idênticas. Todas as medições foram realizadas a $298 \mathrm{~K}$, colocando a sandwich de amostras, mais fonte, num tubo de aço inoxidável, no vazio.

Os espectros de tempo de vida (LT lifetime) foram registrados num sistema de PALS de coincidências rápidas (com cintiladores plásticos de "Pilot-U" e fotomultiplicadores XP 2020), com uma resolução em tempo de 270 ps $[4,5]$. Cada amostra foi medida várias vezes (três a quatro espectros), contendo cada espectro ca. 2,3 × $10^{6}$ contagens. Os espectros de tempo foram analisados pelo programa LT, versão 9 [6].

NO PROCESSO DE INCLUSÃO DE TQ@ $\beta-C D$ FICAM POUCOS ESPAÇOS LIVRES NA CAVIDADE DE $\beta$-CD

Os espectros de tempo foram sujeitos a uma análise com apenas três componentes temporais, i.e., três vidas médias, $\tau_{\mathrm{i}}$, e as respetivas abundâncias relativas (intensidades), $I_{\mathrm{i}}$. Nestas condições, $\tau_{3}$ e $l_{3}$ estão associados com a aniquilação por captação (pick-off) do o-Ps nos volumes livres das amostras de CDs.

Tao e Eldrup [7, 8] propuseram um modelo semi-empírico, a partir da resolução do problema de uma partícula, o Ps, encerrada num potencial esférico de raio $\mathrm{R}_{0}$ e altura infinita, cuja função de onda se sobrepõe à distribuição de eletrões sobre uma calote esférica de raio $\mathrm{R}$. Esse modelo permite estabelecer a seguinte correlação entre o raio do buraco do volume livre, R (em $\AA$ ), e a vida média do o-Ps por pick-off, $\tau_{3}$ (em ns):

$$
\tau_{\mathrm{o}-\mathrm{Ps}}=0,5\left[1-\frac{\mathrm{R}}{\mathrm{R}_{0}}+\frac{1}{2 \pi} \sin \left(\frac{2 \pi \mathrm{R}}{\mathrm{R}_{0}}\right)\right]^{-1}
$$

Aqui $R_{0}=R+\Delta R$, e $\Delta R=1,656 \AA$ representa a espessura de uma camada eletrónica homogénea para a aniquilação pick-off.

Sendo $\mathrm{R}$ o raio médio dos buracos de volume livre, é possível determinar o volume médio dos buracos tomando como $V_{f}=(4 / 3) \pi R^{3}$, grandeza que pode ser calculada a partir da medida do tempo de vida do positrónio. Embora o volume aparente livre integre apenas os locais detetados pelo o-Ps [9], o modelo tem sido utilizado com sucesso em estudos por PALS de numerosas substâncias, incluindo polímeros, membranas [10, 11] e agora as ciclodextrinas [5, 12]. Embora a equação 1 tenha sido obtida para buracos esféricos, o que não é o caso nas CDs, nem tão pouco na maioria das outras substâncias referidas, 0 valor de $\mathrm{R}$ extraído de $\tau_{3}$ pode ser considerado como um raio "médio" e, como tal, é correntemente associado ao "volume livre médio" dos sistemas estudados.

Além dos tempos de vida $\left(\tau_{3}\right)$, e das intensidades $\left(I_{3}\right)$, observados nas amostras de $\beta-C D / T Q$, incluindo $\beta-C D$ pura como referência, a Figura 9 apresenta os raios obtidos com a equação 1 . É de sublinhar o bom acordo dos valores encontrados para $\beta-C D$ pura com resultados obtidos anteriormente [5]. Para a inclusão da timoquinona (TQ), o valor do tempo de vida $\tau_{3}$ diminui com o aumento da concentração de

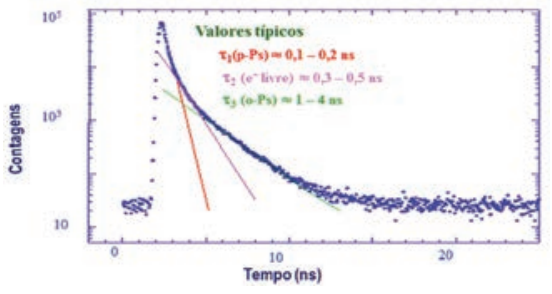

Figura 8 - Espectros de tempo com as três componentes temporais observadas em sistemas homogéneos. Nota-se a presença de duas componentes mais lentas; a presença da componente mais rápida só é evidente após desconvolução com a função de resposta

TQ. No entanto, a diminuição moderada observada até 1:0,75 contrasta com o valor obtido para 1:1. Os volumes livres calculados variam de 71,1 a $60,5 A^{3}$. O parâmetro $I_{3}$ apresenta um decréscimo contínuo com o aumento da fracção molar de TQ; a densidade de buracos, com um valor inicial de $23,35 \%$ para $\beta-C D$ pura é, para a estequiometria de $1: 1$, de apenas 0,41\%. O comportamento de $I_{3}$ está de acordo com a diminuição do volume livre no cone truncado da $\beta-C D$, devido à inclusão de moléculas hóspedes, o que indica que os buracos do cone truncado se tornam totalmente ocupados para a estequiometria de 1:1.

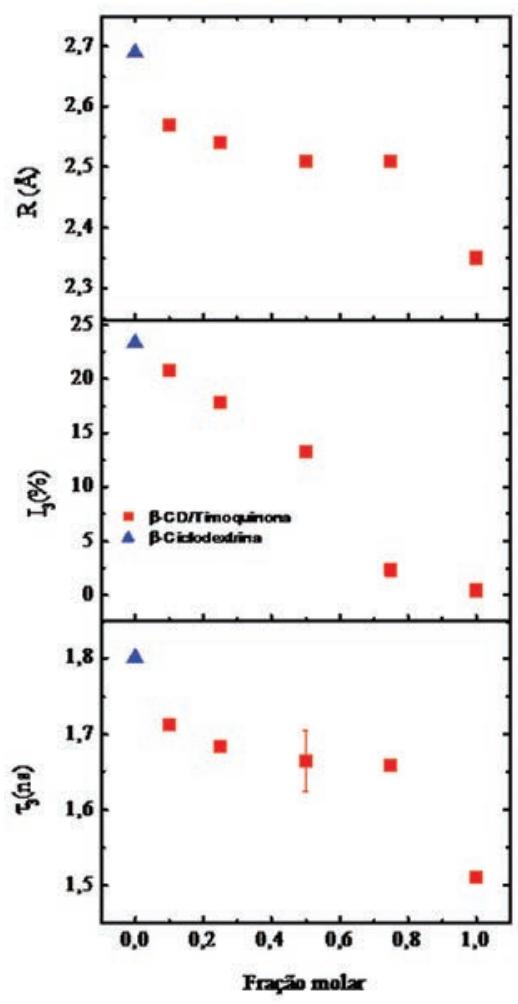

Figura 9 - Parâmetros de PALS (tempos de vida do o-Ps $\left(\tau_{3}\right)$, respetivas intensidades $\left(I_{3}\right) \mathrm{e}$ valores dos raios calculados para os sítios de captura do o-Ps em CDs: $\boldsymbol{\Delta} \beta-\mathrm{CD}, \boldsymbol{\beta} \beta$-CD/TQ 
Além disso, esta última observação está de acordo com resultados de medidas de calorimetria diferencial (DSC - Differential Scanning Calorimetry) e de infravermelho por transformadas de Fourier (FTIR - Fourier Transform Infrared Spectroscopy), e a aplicação do método das variações contínuas (representação de Job) [13, 14]. A técnica de DSC fornece evidência para a inclusão do hóspede, revelando a ausência do pico endotérmico atribuído a fusão da TQ para a maior fração molar. Por sua vez, o espectro de FTIR de $\beta-C D / T h Q$ para 1:1 é muito semelhante ao de $\beta-C D$ pura, o que implica que a TQ seria incluída na cavidade do cone truncado do macrociclo cíclico, $\beta-C D$, e não interposta entre moléculas de $\beta-C D[13,14]$.

O produto de $\tau_{3}$ e $I_{3}$ é uma quantidade interessante, uma vez que está relacionada com o volume livre da amostra $[15,16]$. No presente caso, a inclusão da TQ mostra um decréscimo linear desse produto com o aumento da fração molar. O resultado mostra assim que a estequiometria é de 1:1, uma vez que não temos aí qualquer volume livre.

SERÁ QUE A DIMINUIÇÃO DOS VOLUMES LIVRES NA $\beta$-CD REFLETE O PROCESSO DE INCLUSÃO DA TQ?

O presente estudo de $\beta$-ciclodextrina e seu composto de inclusão pela técnica de PALS fornece uma informação preciosa na determinação do volume livre existente para cada fração molar na determinação da dimensão dos buracos livres e na sua densidade e na confirmação da estequiometria dos complexos. Prova-se, uma vez mais, que a técnica é adequada para a recoIha de informações micro estruturais em tais sistemas.

Deverá contudo dar-se especial atenção à evolução com o tempo decorrido desde o encapsulamento, um aspeto de extrema importância no estudo da permanência do composto que se usa como molécula hóspede "guest". No entanto, a fim de conseguir uma melhor compreensão dos mecanismos de encapsulamento, a utilização de outras técnicas complementares, como as espectroscopias de Raman, de RMN e alargamento Doppler, terá de ser considerada.
Estão já em andamento estudos que visam avaliar o potencial de utilização desses sistemas de inclusão como novos ingredientes funcionais para aplicações em formulações farmacêuticas e/ou em produtos alimentares.

A apresentação deste trabalho sobre o desenvolvimento de um novo potencial ingrediente alimentar funcional é um contributo para a identificação de novos potenciais ingredientes funcionais.

QUAIS SÃO OS OUTROS DESAFIOS PARA A INVESTIGAÇÃO SOBRE Alimentos FunCIONAIS?

Existem vários outros desafios chave para a investigação na área dos alimentos funcionais, como por exemplo a identificação de outros potenciais ingredientes funcionais que poderão fornecer benefícios em termos de saúde e do bem-estar; a identificação de respostas individuais biológicas aos alimentos funcionais; a definição de biodisponibilidade dos ingredientes alimentares funcionais; o desenvolvimento de biomarcadores apropriados a uma ampla gama de parâmetros funcionais; o desenvolvimento da nutrigenómica, da bioinformática, da proteómica, metabolómica e da nanotecnologia no desenvolvimento de alimentos funcionais; a antecipação das necessidades de nutrição personalizada e o papel potencial dos alimentos funcionais. Outros desafios passam também pela realização de estudos de estabilidade dos ingredientes alimentares funcionais durante 0 processamento e na passagem destes através do trato gastrointestinal, assim como estabelecer a Dose Diária Recomendada (DDR), para uma ampla gama de nutrientes, de modo a viabilizar a exploração comercial de ingredientes alimentares funcionais.

\section{NotA}

1 A química para além das moléculas. São estruturas formadas através de ligações do tipo não covalente: de ligação de hidrogénio, interações hidrófobas, interações de van der Waals e interações $\pi-\pi$.

\section{REFERÊNCIAS}

[1] M. Khader, N. Bresgen, P.M. Eckl, Food and Chemical Toxicology 47
(2009) 129-133.

[2] A. Moreira da Silva "Food Products that Help to Promote Human Health", Food, Diet and Health: Past, Present and Future Tendencies, Chapter 4, Nova Science Publishers Inc., Editor Raquel Pinho Ferreira Guiné, pp137193, 2010.

[3] G. Astray, C. Gonzalez-Barreiro, J.C. Mejuto, R. Rial-Otero, J. Simal-Gandara, Hydrocolloids (2009) 25-49.

[4] Y.C. Jean, P.E. Mallon, D.M Schrader, Principles and Applications of Positron and Positronium Chemistry, Word Scentific, London 2003.

[5] M.F. Ferreira Marques, A.M.G. Moreira da Silva, P.M. Gordo, Zs. Kajcsos, Material Science Forum 666 (2011) 99-102.

[6] J. Kansy, Nucl. Instrum. Methods Phys. Res. A 374 (1996) 235-244.

[7] S.J. Tao, Journal of Chemical Physics 56 (1972) 5499-5510.

[8] M. Eldrup, D. Lightbody, J.N. Sherwood, Chemical Physics 63 (1981) 51-58.

[9] F.H.J. Maurer, M. Schmid, Radiation Physics and Chemistry 58 (2000) 509-512.

[10] G. Roudaut, G. Duplâtre, Physical Chemistry Chemical Physics 11 (2009) 9556-9561.

[11] M.F. Ferreira Marques, P.M. Gordo, C. Lopes Gil, A.P. de Lima, D.P. Queiroz, M.N. de Pinho, Zs. Kajcsos, Radiation Physics and Chemistry 76 (2007) 129-133.

[12] M. Roussenova, M. Murith, A. Alam, J. Ubbink, Biomacromolecules 11 (2010) 3237-3247.

[13] T. Cardoso, Development and caractherization of a novel potencial nutraceutical, Relatório de Estágio Profissionalizante, Mestrado em Engenharia Alimentar, Escola Superior Agrária do Instituto Politécnico de Coimbra, 2011.

[14] T. Cardoso, C.I.C. Galhano, M.F. Ferreira Marques, A. Moreira da Silva, Espectroscopia: uma revista internacional 27, 5-6 (2012) 329-336.

[15] G. Dlubek, R. Buchhold, Ch. Hübner; A. Nakldal 32 Macromolecules (2010) 2348-2355.

[16] M.F. Ferreira Marques, P.M. Gordo, S.D. Santos, R.F. Marques, A. Moreira da Silva, Material Science Forum 733 (2013) 88-91. 